INPLASY

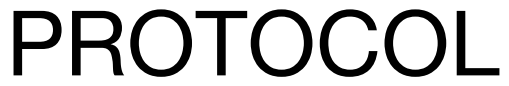

To cite: Huang et al. Vagal maneuvers for supraventricular tachycardia: A systematic review and network metaanalysis. Inplasy protocol 2020110082. doi:

10.37766/inplasy2020.11.0082

Received: 19 November 2020

Published: 19 November 2020

Corresponding author:

Yen Ta Huang

uncleda.huang@gmail.com

Author Affiliation:

Division of Experimental

Surgery, Department of

Surgery, Hualien Tzu Chi

Hospital, Buddhist Tzu Chi

Medical Foundation, Hualien, Taiwan

Support: N/A.

Review Stage at time of this submission: Data analysis.

Conflicts of interest: N/A.

\section{Vagal maneuvers for supraventricular tachycardia: A systematic review and network meta-analysis}

\author{
Huang, EPC1; Chen, $\mathrm{CH}^{2}$; Fan, $\mathrm{CY}^{3}$; Sung, CW4; Lai, PC5; \\ Huang, YT6.
}

Review question / Objective: How about the conversion rate to sinus rhythm at endpoint of studies, initial response, 1minute response and adverse events of all kinds of vagal maneuvers for supraventricular tachycardia?

Condition being studied: Several case-control studies and randomized controlled trials tried to evaluate the efficacy and side effects between different vagal maneuvers. These studies seemly suggested modified Valsalva maneuver as the optimal vagal maneuver for supraventricular tachycardia. However, small number of cases were included in these reports. Based on the principle of evidence-based appraisal, the comparison of effectiveness and adverse effects among all vagal maneuvers remain unclear. We thereby performed a systemic review and a network meta-analysis to investigate all the vagal maneuvers in adult patients with SVT, to identify the effectiveness of treatment, and to report the potential adverse events.

INPLASY registration number: This protocol was registered with the International Platform of Registered Systematic Review and Meta-Analysis Protocols (INPLASY) on 19 November 2020 and was last updated on 15 April 2021 (registration number INPLASY2020110082).

\section{INTRODUCTION}

Review question / Objective: How about the conversion rate to sinus rhythm at endpoint of studies, initial response, 1minute response and adverse events of all kinds of vagal maneuvers for supraventricular tachycardia?
Condition being studied: Several casecontrol studies and randomized controlled trials tried to evaluate the efficacy and side effects between different vagal maneuvers. These studies seemly suggested modified Valsalva maneuver as the optimal vagal maneuver for supraventricular tachycardia. However, small number of cases were 
included in these reports. Based on the principle of evidence-based appraisal, the comparison of effectiveness and adverse effects among all vagal maneuvers remain unclear. We thereby performed a systemic review and a network meta-analysis to investigate all the vagal maneuvers in adult patients with SVT, to identify the effectiveness of treatment, and to report the potential adverse events.

\section{METHODS}

Search strategy: We systematically searched PubMed, Web of Science, Embase, Cochrane Library and Airiti Library to enroll adequate studies by two independent investigators from inception to March 31, 2020. Keywords for search included "supraventricular tachycardia", "Valsalva", "maneuvers", "carotid sinus massage", "ice", "immersion", and "breath holding". Our search strategy aimed to include every clinical trials investigating the use of a non-invasive maneuvers in adult patients with SVT. We systematically searched updatedly to February 28, 2021 to enroll eligible studies.

Participant or population: Patients $\geq 18$ years old with supraventricular tachycardia (SVT).

\section{Intervention: Modified Valsalva maneuver.}

Comparator: Carotid sinus massage, standardized Valsalva maneuver, and other vagal maneuvers.

Study designs to be included: Articles were included if they were case-control studies, involving adult patients $\geq 18$ years old with SVT with comparison of at least two noninvasive maneuvers for rhythm conversion before pharmacologic treatment.

Eligibility criteria: Studies were excluded if they did not meet inclusion criteria, were reviews, case reports or case series, letter to editors, commentaries, or conference abstracts, and those with incorrect study design.
Information sources: We systematically searched PubMed, Web of Science, Embase, Cochrane Library and Airiti Library to enroll adequate studies by two independent investigators from inception to October 31, 2020. Keywords for search included "supraventricular tachycardia", "Valsalva", "maneuvers", "carotid sinus massage", "ice", "immersion", and "breath holding". Our search strategy aimed to include every clinical trials investigating the use of a non-invasive maneuvers in adult patients with SVT. We systematically searched updatedly to February 28, 2021 to enroll eligible studies.

Main outcome(s): The primary outcome was successful conversion to sinus rhythm at initial response, first minute and the endpoint of each studies. The risk of adverse events included hypotension, nausea, dyspnea, arrhythmias, dizziness, or other patient discomforts mentioned by the authors were also analyzed.

Quality assessment / Risk of bias analysis: The risk of bias and internal validity were assessed by two senior authors (Lai PC and Huang YT) independently using the "Risk-of-bias tool 2.0 (RoB 2.0)" for RCTs and the "Risk of Bias in Non-randomized Studies of Interventions (ROBINS-I) tool" developed by the Cochrane collaboration for non-randomized studies.

Strategy of data synthesis: Dichotomous and categorical variables were reported as absolute number and percentages, and continuous variables were reported as mean \pm SD or median (interquartile range).

Subgroup analysis: N/A.

Sensibility analysis: N/A.

Language: No limitation.

Country(ies) involved: Taiwan.

Keywords: vaga I maneuver, supraventricular tachycardia, standard Valsalva maneuvers, modified Valsalva maneuvers, carotid sinus maneuvers, network meta-analysis. 
Contributions of each author:

Author 1 - Edward Pei-Chuan Huang Database search and data extraction, critical analysis, interpretation of the data, and drafting of the manuscript.

Author 2 - Chi-Hsin Chen - Database search and data extraction, critical analysis, interpretation of the data, and drafting of the manuscript.

Author 3 - Cheng-Yi Fan - Database search and data extraction and drafting of the manuscript.

Author 4 - Chih-Wei Sung - Interpretation of the data, revising the manuscript.

Author 5 - Pei-Chun Lai - Double confirming of the enrolled studies and data, concept of the network meta-analysis, statistical analyses, grading of risk of bias and drafting of the manuscript.

Author 6 - Yen-Ta Huang - Double confirming of the enrolled studies and data, concept of the network meta-analysis, statistical analyses, grading of risk of bias and drafting of the manuscript. 